\title{
Stability analysis of a cobweb model with market interactions
}

\author{
Roberto Dieci*(roberto.dieci@unibo.it) \\ Department of Mathematics for Economic and Social Sciences, \\ University of Bologna, Italy \\ Frank Westerhoff (frank.westerhoff@uni-bamberg.de) \\ Department of Economics, University of Bamberg, Germany
}

\begin{abstract}
This paper explores the steady-state properties and the dynamic behavior of a generalization of the classical cobweb model. Under fairly general demand and cost functions, producers form naïve expectations about future prices and select their output so as to maximize expected profits. Unlike the traditional setup, producers have the choice between two markets, and tend to enter that which was more profitable in the recent past. Such a switching process implies time-varying aggregated supply schedules, thus representing a further source of nonlinearity for the dynamics of prices. Analytical investigations and the numerical simulation of a particular case with linear demand and supply indicate that such interactions may destabilize otherwise stable markets and generate complex dynamics.
\end{abstract}

Keywords: cobweb model, interacting markets, bounded rationality, stability, bifurcation analysis

\footnotetext{
${ }^{*}$ Corresponding author. Address: Department of Mathematics for Economic and Social Sciences, University of Bologna, Viale Q. Filopanti 5, I-40126 Bologna, Italy. Phone: +39 0541 434140, Fax: +39 0541434120.
} 


\section{Introduction}

Cobweb models describe the price dynamics in a market of a nonstorable good that takes one time unit to produce. Such a setup is, for instance, typical for agricultural markets. Due to the production lag, producers form price expectations and undertake production decisions one time period ahead, based on current and past experience. Within the early cobweb model of Ezekiel [1], producers simply form naïve expectations, and demand and supply schedules are linear. Despite such a simple setup, this model provides a qualitative explanation for the cyclical tendencies observed in many commodity markets. ${ }^{1}$ Nevertheless, the basic model has only a pedagogical value, and the possible range of dynamic outcomes is basically restricted to either dampened or exploding oscillations around the equilibrium price.

In the last twenty years, the growing popularity of nonlinear dynamics in economic analysis has brought about a renewed interest in cobweb models, and the basic setup has been extended or modified so as to include various nonlinear elements. In particular, Chiarella [6], Day [7] and Hommes [8], [9] consider nonlinear demand and supply curves together with different adaptive expectations schemes. Brock and Hommes [10], Goeree and Hommes [11], Branch [12] and Chiarella and He [13] assume that agents switch between different available prediction rules, depending on certain fitness measures. Risk aversion and time-varying second moment beliefs are introduced into the basic setup by Boussard [14] and Chiarella et al. [15].

By assuming sufficiently general demand and supply functions, the present paper also belongs to this stream of research, albeit extending the model in a different direction. We take into account the fact that producers are able to manufacture different goods. For instance, farmers may decide to expand the production of wheat if they intend to reduce (or abandon) their production of rye. As a result, simple cobweb markets become linked from the supply side. ${ }^{2}$ To make matters as simple as possible, we consider a situation in which producers can choose between one of two markets. The producers' choice, which depends on how profitable the two markets were in the recent past, is updated over time. The more successful market will consequently be selected by more producers than its counterpart. Since the number of producers in a market varies over time, the supply schedule turns out to be state-dependent. Analytical

\footnotetext{
${ }^{1}$ Note that actual commodity price fluctuations are characterized by a strong cyclical component (see, e.g. [2]). Moreover, both empirical evidence $([3],[4])$ and laboratory cobweb experiments ([5]) suggest that agents rely on simple strategies to predict prices.

${ }^{2} \mathrm{~A}$ number of authors have analyzed interdependent cobweb economies for substitute or complement goods, linked from the demand side (e.g. [16], [17]).
} 
and numerical tools prove that this simple nonlinear interaction mechanism has the potential to destabilize otherwise stable stationary states, and to produce complex price dynamics even if the other parts of the cobweb model are specified in a linear manner.

The structure of the paper is as follows. In Section 2, we present a model with two interacting cobweb markets. In Section 3, we reduce the model to a 4-dimensional discrete-time nonlinear dynamical system, derive analytical results about the steady state and its local stability properties (3.1), and provide a numerical example of complex price fluctuations around an unstable steady state (3.2). Section 4 concludes the paper.

\section{Model}

We consider two markets, called markets $X$ and $Z$. At the beginning of each period, producers select the market they wish to enter. Given a fixed number $N$ of producers, the proportions entering markets $X$ and $Z$ at time step $t$ are denoted as $W_{X, t}$ and $W_{Z, t}=1-W_{X, t}$, respectively. An individual producer either supplies quantity $S_{X, t}$ or $S_{Z, t}$. Hence, the total supply in the two markets is $N W_{X, t} S_{X, t}, N W_{Z, t} S_{Z, t}$, respectively. Market clearing occurs in every period, implying that

$$
D_{X, t}=N W_{X, t} S_{X, t}, \quad D_{Z, t}=N W_{Z, t} S_{Z, t},
$$

where $D_{X, t}$ and $D_{Z, t}$ denote the demand for goods $X$ and $Z$, respectively. All other parts of the model are specified by extending the classical cobweb setup, based on the assumption of profit-maximizing producers ${ }^{3}$ endowed with naïve expectations, to fairly general demand and cost functions. Let us now describe our assumptions in detail.

Consumer demand for each good is a strictly decreasing function of its own current market price $\left(P_{X, t}\right.$ or $\left.P_{Z, t}\right)$

$$
D_{X, t}=D_{X}\left(P_{X, t}\right), \quad D_{Z, t}=D_{Z}\left(P_{Z, t}\right)
$$

with $D_{X}^{\prime}, D_{Z}^{\prime}<0$.

The producers' supply is a strictly increasing function of the expected price. Let us denote by $C_{X}\left(S_{X, t}\right)$ and $C_{Z}\left(S_{Z, t}\right)$ the cost functions of goods $X$ and $Z$, respectively, and assume positive and strictly increasing marginal costs, $C_{X}^{\prime}, C_{Z}^{\prime}>0, C_{X}^{\prime \prime}, C_{Z}^{\prime \prime}>0$. For each good (here we omit subscripts $X$ and $Z$ ), the optimal supply $S_{t}$ for period $t$ is determined in period $t-1$ by solving

\footnotetext{
${ }^{3}$ A related study ([18]) assumes that producers maximize expected utility of wealth, thus focusing explicitly on the role of risk aversion.
} 
$\max _{S_{t}} \mathbb{E}_{t-1}\left(\pi_{t}\right)$, i.e.

$$
\max _{S_{t}}\left[S_{t} \mathbb{E}_{t-1}\left(P_{t}\right)-C\left(S_{t}\right)\right]
$$

where $\pi_{t}=P_{t} S_{t}-C\left(S_{t}\right)$ represents profit ${ }^{4}$ in period $t$, and $\mathbb{E}_{t-1}$ denotes the conditional expectation operator. From the first-order condition of (3), under naïve expectations ${ }^{5}, \mathbb{E}_{t-1}\left(P_{t}\right)=P_{t-1}$, the supply of a single producer is therefore either $S_{X, t}$ or $S_{Z, t}$, where

$$
S_{X, t}=G_{X}\left(P_{X, t-1}\right), \quad S_{Z, t}=G_{Z}\left(P_{Z, t-1}\right)
$$

and where $G_{X}(\cdot):=\left(C_{X}^{\prime}\right)^{-1}(\cdot), G_{Z}(\cdot):=\left(C_{Z}^{\prime}\right)^{-1}(\cdot)$ denote the (strictly increasing) inverse marginal cost functions. The market clearing conditions (1) thus yield the laws of motion of the two prices, i.e.

$$
P_{X, t}=D_{X}^{-1}\left(N W_{X, t} G_{X}\left(P_{X, t-1}\right)\right),
$$

and

$$
P_{Z, t}=D_{Z}^{-1}\left(N W_{Z, t} G_{Z}\left(P_{Z, t-1}\right)\right)
$$

where $D_{X}^{-1}(\cdot)$ and $D_{Z}^{-1}(\cdot)$ denote the (strictly decreasing) inverse demand functions.

Obviously, in the case of constant proportions, $W_{X, t}=\bar{W}_{X}, W_{Z, t}=\bar{W}_{Z}=1-\bar{W}_{X}$, the two markets evolve independently, each driven by a first-order linear difference equation. In this case, steady state prices $\bar{P}_{X}$ and $\bar{P}_{Z}$ are determined implicitly as follows

$$
\bar{P}_{X}=D_{X}^{-1}\left(N \bar{W}_{X} G_{X}\left(\bar{P}_{X}\right)\right), \quad \bar{P}_{Z}=D_{Z}^{-1}\left(N\left(1-\bar{W}_{X}\right) G_{Z}\left(\bar{P}_{Z}\right)\right)
$$

For each good ${ }^{6}$, denote by $\left(P^{l}, P^{u}\right), 0 \leq P^{l}<P^{u} \leq+\infty$, a price interval over which both demand and supply functions, $D(P)$ and $G(P)$, are strictly positive and satisfy the above assumed monotonicity properties. Assume also:

$$
\lim _{P \rightarrow P^{l}}[D(P)-N G(P)]>0, \quad \lim _{P \rightarrow P^{u}} D(P)=0 .
$$

Together with continuity and strict monotonicity of $D(P)$ and $G(P)$, conditions (8) ensure that a unique solution $\bar{P} \in\left(P^{l}, P^{u}\right)$ to each of equations (7) exists for any $\bar{W}, 0<\bar{W}<1$, that is, a

\footnotetext{
${ }^{4}$ Profit $\pi_{t}$ is regarded as a random variable in period $t-1$.

${ }^{5}$ Note that naïve expectations entail a supply response lag, i.e. the supply in period $t$ depends on the realized price in period $t-1$.

${ }^{6}$ Again, we omit subscripts $X$ and $Z$.
} 
unique steady state exists for any fixed distribution of producers over the two markets. ${ }^{7}$

The unique steady state of each independent market is locally asymptotically stable under a certain relation between the slopes of its demand and total supply schedules. Denoting the steady-state aggregate supply of goods $X$ and $Z$, respectively, by $\bar{S}_{X}^{A}:=N \bar{W}_{X} G_{X}\left(\bar{P}_{X}\right), \bar{S}_{Z}^{A}:=$ $N\left(1-\bar{W}_{X}\right) G_{Z}\left(\bar{P}_{Z}\right)$, the local asymptotic stability of steady-state prices of the two markets requires

$$
\left|\left(D_{X}^{-1}\right)^{\prime}\left(\bar{S}_{X}^{A}\right) N \bar{W}_{X} G_{X}^{\prime}\left(\bar{P}_{X}\right)\right|<1, \quad\left|\left(D_{Z}^{-1}\right)^{\prime}\left(\bar{S}_{Z}^{A}\right) N\left(1-\bar{W}_{X}\right) G_{Z}^{\prime}\left(\bar{P}_{Z}\right)\right|<1 .
$$

Since $\left(D_{X}^{-1}\right)^{\prime}\left(\bar{S}_{X}^{A}\right)=1 / D_{X}^{\prime}\left(\bar{P}_{X}\right),\left(D_{Z}^{-1}\right)^{\prime}\left(\bar{S}_{Z}^{A}\right)=1 / D_{Z}^{\prime}\left(\bar{P}_{Z}\right)$ by inverse function rule, the local stability conditions (9) take the familiar form in terms of ratios between slopes

$$
\left|\frac{N \bar{W}_{X} G_{X}^{\prime}\left(\bar{P}_{X}\right)}{D_{X}^{\prime}\left(\bar{P}_{X}\right)}\right|<1, \quad\left|\frac{N\left(1-\bar{W}_{X}\right) G_{Z}^{\prime}\left(\bar{P}_{Z}\right)}{D_{Z}^{\prime}\left(\bar{P}_{Z}\right),}\right|<1 .
$$

As is well known from the classical cobweb setup, converging price paths display temporary up-and-down oscillations around the long-run equilibrium price.

Time-varying, state-dependent proportions of producers, however, result in endogenous interactions between markets $X$ and $Z$. The producers are boundedly rational in the sense that they tend to select the market which would have been more profitable for them in the last period. Assuming a high number of producers, fractions $W_{X, t}$ and $W_{Z, t}$ can be determined via a discrete choice model (see, e.g. [10]), i.e.

$$
W_{X, t}=\frac{\exp \left(f \pi_{X, t-1}\right)}{\exp \left(f \pi_{X, t-1}\right)+\exp \left(f \pi_{Z, t-1}\right)}, \quad W_{Z, t}=\frac{\exp \left(f \pi_{Z, t-1}\right)}{\exp \left(f \pi_{X, t-1}\right)+\exp \left(f \pi_{Z, t-1}\right)},
$$

where $\pi_{X, t-1}=P_{X, t-1} S_{X, t-1}-C_{X}\left(S_{X, t-1}\right), \pi_{Z, t-1}=P_{Z, t-1} S_{Z, t-1}-C_{Z}\left(S_{Z, t-1}\right)$ denote realized profits of the two markets in period $t-1$. Parameter $f \geq 0$ is called the intensity of choice, and measures how sensitive the mass of producers is to selecting the most profitable market. For $f=0$, the agents do not observe any profit differentials between the two markets. As a result, $W_{X, t}=W_{Z, t}=\bar{W}=\frac{1}{2}$ for any $t$, and we obtain a fixed-proportion model, with producers evenly distributed across two independent markets. On the other hand, the higher $f$ is, the larger the proportion of producers who select the market that performed better in the previous period, for any observed profit differential. In the extreme case $f \rightarrow \infty$, all producers in each period enter

\footnotetext{
${ }^{7}$ This is the case, for instance, for the linear specifications used in the numerical simulation in Section 3.2.
} 
the market with the higher realized profit in the previous period. ${ }^{8}$

Of course, both naïve price expectations and the 'logistic' choice of a market are very specific (and simplifying) assumptions. Nonetheless, the latter represents a very common assumption in the literature on evolutionary learning (see, e.g. [10], and references therein), whereas naïve expectations are assumed within the present paper only in order to stick to the classical cobweb setup, and to focus on the mere effect of interaction. A further simplification in our setup concerns the cost of switching between markets, which is assumed to be zero here for reasons of analytical tractability. ${ }^{9}$

\section{Dynamical system}

By substituting (11) into (5) and (6), one obtains a system of two nonlinear second-order difference equations in the prices. ${ }^{10}$ The system can be rewritten as a $4-D$ dynamical system in the state variables $P_{X}, P_{Z}, S_{X}, S_{Z}$. Let us introduce the difference of proportions ${ }^{11} \Omega_{t}$ $:=W_{X, t}-W_{Z, t}$. This can be rewritten as $\Omega_{t}=\tanh \left[\frac{f}{2}\left(\pi_{X, t-1}-\pi_{Z, t-1}\right)\right]$, with $-1<\Omega_{t}<1$, where $\Omega_{t} \rightarrow 1$ corresponds to $W_{X, t} \rightarrow 1$ and $\Omega_{t} \rightarrow-1$ corresponds to $W_{Z, t} \rightarrow 1$. The resulting $4-D$ nonlinear dynamical system is thus the following:

$$
\begin{gathered}
P_{X, t}=F_{X}\left(P_{X, t-1}, P_{Z, t-1}, S_{X, t-1}, S_{Z, t-1}\right)=D_{X}^{-1}\left(\frac{N}{2}\left(1+\Omega_{t}\right) G_{X}\left(P_{X, t-1}\right)\right) \\
P_{Z, t}=F_{Z}\left(P_{X, t-1}, P_{Z, t-1}, S_{X, t-1}, S_{Z, t-1}\right)=D_{Z}^{-1}\left(\frac{N}{2}\left(1-\Omega_{t}\right) G_{Z}\left(P_{Z, t-1}\right)\right) \\
S_{X, t}=G_{X}\left(P_{X, t-1}\right) \\
S_{Z, t}=G_{Z}\left(P_{Z, t-1}\right)
\end{gathered}
$$

\footnotetext{
${ }^{8}$ An alternative interpretation of proportions $W_{X, t}$ and $W_{Z, t}$ could be in terms of composition of the supply by one representative farmer. This producer would then only adjust the output mix without moving completely across markets.

${ }^{9}$ Note that high switching costs might have a considerable impact on the dynamics. With regard to such costs, parameter $f$ could be interpreted not only as the 'intensity of choice' but also, in terms of the original 'discrete choice' model, as a quantity inversely related to the variance of the distribution of the switching cost (see, e.g. [19]). For instance, a low value of $f$ would imply that a substantial fraction of producers changes only if the profit differential is quite high as compared to the average cost of switching. We thank a referee for pointing this out to us.

${ }^{10}$ Note that $\pi_{X, t-1}\left(\pi_{Z, t-1}\right)$ depends on both $P_{X, t-1}$ and $P_{X, t-2}\left(P_{Z, t-1}\right.$ and $\left.P_{Z, t-2}\right)$.

${ }^{11}$ The same change of variable is used, e.g. in [10]. Note that from $W_{Z, t}=1-W_{X, t}$, it follows that $W_{X, t}=$ $\left(1+\Omega_{t}\right) / 2, W_{Z, t}=\left(1-\Omega_{t}\right) / 2$, and that $\Omega_{t}=2 W_{X, t}-1=1-2 W_{Z, t}$.
} 
where

$$
\begin{gathered}
\Omega_{t}=\Omega\left(P_{X, t-1}, P_{Z, t-1}, S_{X, t-1}, S_{Z, t-1}\right)=\tanh \left[\frac{f}{2}\left(\pi_{X, t-1}-\pi_{Z, t-1}\right)\right] \\
\pi_{X, t-1}=P_{X, t-1} S_{X, t-1}-C_{X}\left(S_{X, t-1}\right), \quad \pi_{Z, t-1}=P_{Z, t-1} S_{Z, t-1}-C_{Z}\left(S_{Z, t-1}\right) .
\end{gathered}
$$

The Propositions in the following subsection characterize the (unique) steady state of the dynamical system (12)-(15), in terms of the stationary distribution of producers. Moreover, they establish a number of analytical results on local asymptotic stability, and analyze the impact of the assumed interaction mechanism, compared to the case of markets in isolation.

\subsection{Steady state and local stability}

We use an overbar to denote steady-state quantities. In particular, $\bar{\Omega}:=\bar{W}_{X}-\bar{W}_{Z}$ represents the stationary "distribution" of producers across markets. The existence and uniqueness of the steady state of the dynamic model (12)-(15), as well as its characterization in terms of distribution of producers, are stated in the following

\section{Proposition 1}

(i) The dynamical system (12)-(15) admits a unique steady state $\mathbf{q}^{*}=\left(\bar{P}_{X}, \bar{P}_{Z}, \bar{S}_{X}, \bar{S}_{Z}\right)$.

(ii) The steady-state "distribution" of producers across markets, $\bar{\Omega}$, is positive (negative) and increases (decreases) with parameter $f$ if and only if the difference between the steady-state profits of the independent markets $X$ and $Z$, in the case of no switching $(f=0)$, is positive (negative).

Proof: see Appendix A

According to Proposition 1, the market that will attract a higher proportion of producers in equilibrium is that which would be more profitable in the absence of interaction (i.e. in the case $f=0$ ). This confirms our intuition. Furthermore, the producers' steady-state proportion in such a market depends positively on the intensity of choice $f$. Note that, even when adopting very simple specifications of demand and cost functions, the steady state cannot be computed analytically, as is also clear from the proof in Appendix A.

The next Proposition and the subsequent Corollaries provide general results on the stability properties of the steady state and highlight further connections to the case of isolated markets. 


\section{Proposition 2}

(i) The steady state $\mathbf{q}^{*}$ of the dynamical system (12)-(15) is locally asymptotically stable (LAS) in the region of the space of parameters where the following inequality is satisfied

$$
\gamma_{X}\left(1+f \delta_{X}\right)+\gamma_{Z}\left(1+f \delta_{Z}\right)<\min \left[1+\gamma_{X} \gamma_{Z}\left(1+f\left(\delta_{X}+\delta_{Z}\right)\right), 2\right],
$$

where

$$
\begin{gathered}
\gamma_{X}:=\left|\frac{N(1+\bar{\Omega}) G_{X}^{\prime}\left(\bar{P}_{X}\right)}{2 D_{X}^{\prime}\left(\bar{P}_{X}\right)}\right|, \quad \gamma_{Z}:=\left|\frac{N(1-\bar{\Omega}) G_{Z}^{\prime}\left(\bar{P}_{Z}\right)}{2 D_{Z}^{\prime}\left(\bar{P}_{Z}\right),}\right|, \\
\delta_{X}:=\frac{(1-\bar{\Omega}) \bar{S}_{X}^{2}}{2 G_{X}^{\prime}\left(\bar{P}_{X}\right)}, \quad \delta_{Z}:=\frac{(1+\bar{\Omega}) \bar{S}_{Z}^{2}}{2 G_{Z}^{\prime}\left(\bar{P}_{Z}\right)},
\end{gathered}
$$

and the loss of stability can only occur via a Flip bifurcation.

(ii) For parameters in the 'stability region' (18), the following conditions are necessarily satisfied

$$
\begin{gathered}
\left|\frac{N(1+\bar{\Omega}) G_{X}^{\prime}\left(\bar{P}_{X}\right)}{2 D_{X}^{\prime}\left(\bar{P}_{X}\right)}+\frac{f}{2} \frac{N\left(1-\bar{\Omega}^{2}\right) \bar{S}_{X}^{2}}{2 D_{X}^{\prime}\left(\bar{P}_{X}\right)}\right|<1, \\
\left|\frac{N\left(1-\bar{\Omega}^{\prime}\right) G_{Z}^{\prime}\left(\bar{P}_{Z}\right)}{2 D_{Z}^{\prime}\left(\bar{P}_{Z}\right)}+\frac{f}{2} \frac{N\left(1-\bar{\Omega}^{2}\right) \bar{S}_{Z}^{2}}{2 D_{Z}^{\prime}\left(\bar{P}_{Z}\right)}\right|<1 .
\end{gathered}
$$

\section{Proof: see Appendix B}

The second part of Proposition 2 makes it possible to compare the stability domain (18) of the complete model with two 'reference' cases, both characterized by a fixed distribution of producers across markets. In the first case (discussed in Corollary 3), the fixed distribution $\left(\bar{W}_{X}, \bar{W}_{Z}\right)$ is exactly the same as the steady-state distribution of the model with endogenously varying fractions. ${ }^{12}$ The second case (Corollary 4 ) is obtained by setting $f=0$ in the dynamical system (12)-(15), which results in a model with no switching and producers splitting evenly between markets $X$ and $Z$.

Corollary 3 For a given $f>0$, condition (18) is more restrictive, for the slopes of the demand and (individual) supply curves in each market, than the local stability conditions of the corresponding fixed-proportion model.

Proof: see Appendix C

\footnotetext{
${ }^{12}$ We thank one of the referees for encouraging us to compare stability conditions of the complete model with the constant proportion case in Corollary 3.
} 
Corollary 4 Let $X(Z)$ be the market with higher steady-state profit under the no-switching case $(f=0)$. Then, under broad conditions on the slopes of demand and supply curves, the stability of the complete model with switching $(f>0)$ requires the stability of the 'independent' market $X(Z)$ in the absence of switching.

Proof: see Appendix C

Corollaries 3 and 4 deserve further comments. As already mentioned, Corollary 3 compares stability results stated in Proposition 2 with the case of two decoupled markets with fixed proportions of suppliers equal to those who are active at the steady state of the coupled model. Such a comparison is quite natural and provides an explicit intuition of why two markets that are stable when considered in isolation can be destabilized once suppliers are allowed to switch between them. In the standard cobweb model (with no switching), an increase in the market clearing price triggers an increase in supply by farmers who are active in that market, which, in turn, decreases the market clearing price. As a consequence, the supply in the next period declines, leading to an immediate increase in the price, and so on. For suitable ranges of the slopes of demand and supply schedules, these oscillating prices converge to a steady state. Things may be different if farmers can switch between the two markets. If the system is perturbed from the steady state by increasing the price in one market, this does not only increase the supply of the active farmers, but also attracts new producers into that market. An otherwise stable cobweb market may thus be destabilized because supply becomes more sensitive to market prices. This additional effect is captured, e.g. for market $X$, by the second term on the left-hand side in equation (21), whereas the first term is related to the change in supply by active farmers, as in the fixed-proportions model (see equation (10)).

Corollary 4 focuses on the role of parameter $f$, and compares stability condition (18) of the full model (with $f>0$ ) with the limiting case of zero intensity of switching $(f=0)$. The latter results in two decoupled markets with uniform distribution of producers $(\bar{\Omega}=0)$, and stability condition (18) (as well as necessary condition (21)-(22)) reduces to

$$
\left|\frac{N G_{X}^{\prime}\left(\bar{P}_{X}^{0}\right)}{2 D_{X}^{\prime}\left(\bar{P}_{X}^{0}\right)}\right|<1, \quad\left|\frac{N G_{Z}^{\prime}\left(\bar{P}_{Z}^{0}\right)}{2 D_{Z}^{\prime}\left(\bar{P}_{Z}^{0}\right)}\right|<1,
$$


where $\bar{P}_{X}^{0}$ and $\bar{P}_{Z}^{0}$ denote steady state prices in the case $f=0$, implicitly defined by equations (33) in Appendix A. ${ }^{13}$ Comparison with such a case is less straightforward than before, because an increase of parameter $f$ changes both the location and the stability properties of the steady state. However, also from this perspective, the necessary condition (21)-(22) highlights the possible destabilizing impact of the assumed interaction mechanism. Consider, for instance, the case of linear demand and supply (developed in detail in the next section), where $\left|G_{X}^{\prime} / D_{X}^{\prime}\right|$ and $\left|G_{Z}^{\prime} / D_{Z}^{\prime}\right|$ are constant. ${ }^{14}$ According to Corollary 4, in order for the steady state of the complete model to be stable, the slopes of demand and (individual) supply curves in markets $X$ and $Z$ must be such that at least the 'more profitable' market is stable, when considered in isolation. Moreover, if, e.g. $X$ is the market with higher steady-state profit in the absence of interaction, condition (21) is more restrictive, for the slopes of demand and supply curves in market $X$, than stability condition (23) for the isolated market $X .{ }^{15}$ A similar reasoning applies to market $Z$. Finally, numerical simulation reveals that the necessary stability condition (21)-(22) will be violated whenever (23) holds, but the ratios $\left|G_{X}^{\prime} / D_{X}^{\prime}\right|$ and $\left|G_{Z}^{\prime} / D_{Z}^{\prime}\right|$ are large enough, and this occurs irrespective of which of the two markets is more profitable at the steady state in isolation. This is often the case even with low values of such ratios, as shown in the next section. This fact is obviously related to the impact of the switching parameter $f>0$.

Overall, our results on local stability show that interactions between cobweb markets, arising when agents are allowed to select the most profitable alternative, cannot stabilize two otherwise unstable markets. Rather, under a wide range of circumstances, they tend to destabilize otherwise stable markets. This point is further developed in the following section, where we carry out a numerical example with two connected 'linear' cobweb markets.

\subsection{Interactions and price fluctuations}

The example proposed and discussed in this section is based on the following linear specification of the demand functions

$$
D_{X, t}=\left(a_{X}-P_{X, t}\right) / b_{X}, \quad D_{Z, t}=\left(a_{Z}-P_{Z, t}\right) / b_{Z},
$$

\footnotetext{
${ }^{13}$ Obviously, conditions (23) are nothing but conditions (10) with $\bar{W}_{X}=\bar{W}_{Z}=1 / 2$.

${ }^{14}$ The following discussion applies, however, to far more general cases. See Appendix C.

${ }^{15}$ This results directly from a comparison of $(23)$ with $(21)$ and the fact that $\bar{\Omega}>0$ in this case. See also Appendix C.
} 
where $a_{X}, a_{Z}, b_{X}, b_{Z}>0$. It also assumes quadratic cost functions, i.e.

$$
C_{X}\left(S_{X}\right)=c_{X} S_{X}+\frac{d_{X}}{2} S_{X}^{2}, \quad C_{Z}\left(S_{Z}\right)=c_{Z} S_{Z}+\frac{d_{Z}}{2} S_{Z}^{2}
$$

where $c_{X}, c_{Z} \geq 0, d_{X}, d_{Z}>0$, so that (individual) supply curves turn out to be linear, too, and are expressed as

$$
S_{X, t}=\left(P_{X, t-1}-c_{X}\right) / d_{X}, \quad S_{Z, t}=\left(P_{Z, t-1}-c_{Z}\right) / d_{Z}
$$

In the following, we assume $a_{X}>c_{X}, a_{Z}>c_{Z}$. Demand and supply curves of the two goods are thus strictly positive (and strictly monotonic) over the price ranges $c_{X}<P_{X}<a_{X}, c_{Z}<$ $P_{Z}<a_{Z}$, respectively. Based on the discussion of the fixed-proportion case in Section 2, one can check that a unique steady state price $\bar{P}_{X} \in\left(c_{X}, a_{X}\right), \bar{P}_{Z} \in\left(c_{Z}, a_{Z}\right)$ exists in market $X$, $Z$, respectively, for any fixed distribution of producers over the two markets.

Turning to the model with state-dependent proportions, by setting $g_{X}:=\left(N b_{X}\right) / 2, g_{Z}:=$ $\left(N b_{Z}\right) / 2$, the laws of motion (12)-(13) for prices are specified as follows

$$
P_{X, t}=\frac{a_{X} d_{X}-g_{X}\left(1+\Omega_{t}\right)\left(P_{X, t-1}-c_{X}\right)}{d_{X}}, \quad P_{Z, t}=\frac{a_{Z} d_{Z}-g_{Z}\left(1-\Omega_{t}\right)\left(P_{Z, t-1}-c_{Z}\right)}{d_{Z}} .
$$

The dynamical system thus consists of equations (25) and (24), where

$$
\begin{aligned}
\Omega_{t} & =\tanh \left\{\frac{f}{2}\left[\pi_{X, t-1}-\pi_{Z, t-1}\right]\right\} \\
& =\tanh \left\{\frac{f}{2}\left[\left(P_{X, t-1}-c_{X}\right) S_{X, t-1}-\frac{d_{X}}{2} S_{X, t-1}^{2}-\left(P_{Z, t-1}-c_{Z}\right) S_{Z, t-1}+\frac{d_{Z}}{2} S_{Z, t-1}^{2}\right]\right\} .
\end{aligned}
$$

Simple computations provide the coordinates $\bar{P}_{X}, \bar{P}_{Z}, \bar{S}_{X}$, and $\bar{S}_{Z}$ of the unique steady state, as well as steady-state profits, namely

$$
\begin{gathered}
\bar{P}_{X}=\frac{a_{X} d_{X}+g_{X}(1+\bar{\Omega}) c_{X}}{d_{X}+g_{X}(1+\bar{\Omega})}, \quad \bar{P}_{Z}=\frac{a_{Z} d_{Z}+g_{Z}(1-\bar{\Omega}) c_{Z}}{d_{Z}+g_{Z}(1-\bar{\Omega})} \\
\bar{S}_{X}=\frac{\bar{P}_{X}-c_{X}}{d_{X}}=\frac{a_{X}-c_{X}}{d_{X}+g_{X}(1+\bar{\Omega})}, \quad \bar{S}_{Z}=\frac{\bar{P}_{Z}-c_{Z}}{d_{Z}}=\frac{a_{Z}-c_{Z}}{d_{Z}+g_{Z}(1-\bar{\Omega})} \\
\bar{\pi}_{X}=\frac{d_{X}\left(a_{X}-c_{X}\right)^{2}}{2\left(d_{X}+g_{X}(1+\bar{\Omega})\right)^{2}}, \quad \bar{\pi}_{Z}=\frac{d_{Z}\left(a_{Z}-c_{Z}\right)^{2}}{2\left(d_{Z}+g_{Z}(1-\bar{\Omega})\right)^{2}}
\end{gathered}
$$


where steady-state 'distribution', $\bar{\Omega}$, is defined implicitly by

$$
\bar{\Omega}=\tanh \left\{\frac{f}{2}\left[\frac{d_{X}\left(a_{X}-c_{X}\right)^{2}}{2\left(d_{X}+g_{X}(1+\bar{\Omega})\right)^{2}}-\frac{d_{Z}\left(a_{Z}-c_{Z}\right)^{2}}{2\left(d_{Z}+g_{Z}(1-\bar{\Omega})\right)^{2}}\right]\right\} .
$$

Our simulation analysis ${ }^{16}$ is based on a parameter selection outside the stability domain (18). It illustrates our analytical results and shows that the model can produce complex price movements. These are driven by both the basic cobweb mechanism and the nonlinear switching behavior of the producers. Parameters are set as follows: $a_{X}=a_{Z}=20, c_{X}=c_{Z}=0, d_{X}=d_{Z}=10$, $g_{X}=1.5, g_{Z}=5, f=0.375$. In the absence of interactions (i.e. if $f=0, \bar{\Omega}=0$ ), the steadystate profits of the independent markets would be $\bar{\pi}_{X}^{0} \simeq 15.123, \bar{\pi}_{Z}^{0} \simeq 8.889$, respectively. It then follows from Proposition 1 that $\bar{\Omega}>0$ (i.e. $\bar{W}_{X}>50 \%$ ) at the steady state for any $f>0$, and that $\bar{\Omega}$ (as well as $\bar{W}_{X}$ ) is an increasing function of $f$. Note also that

$$
\left|\frac{N}{2} \frac{G_{X}^{\prime}\left(\bar{P}_{X}^{0}\right)}{D_{X}^{\prime}\left(\bar{P}_{X}^{0}\right)}\right|=g_{X} / d_{X}=0.15, \quad\left|\frac{N}{2} \frac{G_{Z}^{\prime}\left(\bar{P}_{Z}^{0}\right)}{D_{Z}^{\prime}\left(\bar{P}_{Z}^{0}\right)}\right|=g_{Z} / d_{Z}=0.5,
$$

i.e. in the case of no switching $(f=0)$, the steady states of the two independent markets would both be globally asymptotically stable. However, the steady state of the coupled system is LAS only for $f<f_{\text {Flip }} \simeq 0.093311$ (where $f_{\text {Flip }}$ denotes the Flip-bifurcation value for the intensity of choice parameter), as can be computed numerically from (18). Beyond this threshold, interactions destabilize the cobweb markets via a supercritical ${ }^{17}$ Flip bifurcation.

For $f=0.375$, the first three panels of figure 1 show time series for the price in market $X$, the price in market $Z$, and the proportion of producers in market $X$, respectively. As can be seen, prices fluctuate in both markets in an intricate manner around their long-run equilibrium values; the same holds for the producers' distribution across markets.

\section{Figure 1 goes about here}

In a stylized way, the dynamics evolves as follows. Suppose that some producers switch from the less to the more profitable market. In the less profitable market, the total supply decreases and the price increases. In the other market, the opposite occurs: the total supply increases and

\footnotetext{
${ }^{16}$ The analysis of the system is obviously restricted to 'feasible' orbits, i.e. such that $a_{X}>P_{X, t}>c_{X}$, $a_{Z}>P_{Z, t}>c_{Z}$ for any $t$.

${ }^{17}$ The existence of an attracting two-cycle just outside the stability domain, when $f$ is close to the Flipbifurcation value, can be detected numerically and is also revealed by the bifurcation diagrams in figure 2. Thus we claim numerical evidence about the supercritical nature of the Flip bifurcation.
} 
the price decreases. Combined, these two effects may reverse the profit differential again, causing some producers to stream back to the other market. This pattern may repeat itself, albeit in a complicated manner. Under this particular parameter selection, price movements occur along an apparently strange ${ }^{18}$ attractor: the bottom panels of figure 1 represent the projections of the attractor in planes $P_{X}, P_{Z}$ and $P_{X}, S_{X}$, respectively.

The producers' sensitivity to profit differentials, i.e. the intensity of choice parameter $f$, plays a crucial role in driving the evolution of prices: higher $f$ in general increases the amplitude of the fluctuations of prices and distribution of producers across markets, and determines a transition to complex behavior. This is revealed by the bifurcation diagrams given in figure 2, which display the typical period-doubling bifurcation sequence leading to chaotic dynamics. Moreover, starting from about $f=0.578$ a period-three cycle emerges. The nature of this cycle highlights the extreme effects that are brought about by the reversal of profit differentials, when parameter $f$ is large enough: from the second panel of figure 2 we observe that, at two of the three periodic points, the proportion of producers in market $X$ is about $100 \%$, whereas at the remaining point almost all producers have entered market $Z$. This implies that basically all market participants switch from one market to the other within one time step. One may argue that this is not very realistic, i.e. one may conclude that $f$ is presumably not larger than 0.7 in reality, at least for demand and supply parameters similar to those used in our example. Additional simulations (not displayed in the paper) reveal that the period-three cycle also remains intact for much larger values of $f$.

\section{Figure 2 goes about here}

The numerical example illustrated in figures 1 and 2 can be regarded as representative of the dynamic behavior of the model and its dependence on the 'intensity of choice' $f$ : qualitatively similar results can be easily obtained under a variety of configurations for the parameters, and under different specifications of demand and supply functions.

\footnotetext{
${ }^{18}$ In fact, the phase space representation in figure 1 only allows us to conclude that the attractor 'appears' to be strange. We have, however, estimated the Lyapunov exponent and the correlation dimension for this particular simulation run. The Lyapunov exponent is 0.45 and the correlation dimension is 1.23. This strengthens numerical evidence for chaos and strange attractors.
} 


\section{Concluding remarks}

If the price of a commodity decreases, the cobweb scenario predicts that suppliers will reduce their output. However, it does not explore what they will do in such a case as an alternative. Motivated by this observation, this paper investigates an extension of the basic cobweb setup, where it is assumed that in each period producers can select between one of two products (markets), based on realized profits. If a market was relatively profitable, it attracts more producers and the total supply increases. As a consequence, interactions arise between markets for the two products, which may lead to a nonlinear system even starting from the linear cobweb model. In order to focus on the role played by such interactions, we stick to the classical cobweb world as far as possible, by assuming downward-sloped demand curves and strictly increasing (individual) supply schedules, as well as naïve expectations. To model interactions, we introduce a 'logistic' switching mechanism, commonly adopted in the literature on evolutionary learning. Analytical investigation and numerical simulation of the model indicate that such market interactions add to the cyclical component of commodity prices represented by the classical cobweb behavior. Such interactions can destabilize otherwise globally/locally stable equilibria of two cobweb markets considered in isolation. Moreover, complex dynamic scenarios may emerge, even in the case where original independent markets behave linearly, particularly when agents react more sensitively to profit differentials.

The framework provided by this model could be developed in a number of directions. First, it could be adopted to investigate interdependencies on the demand side ${ }^{19}$. For instance, in the case of more general demand functions with positive cross price elasticities (substitute goods), an increase in the supply of one commodity would decrease prices of both commodities and may therefore dampen the interaction effect somehow. It would then be interesting to assess the net effect of demand and supply interdependencies.

Second, the possible impact of the costs of switching resources from one market to the other should be taken into account. This could be done by directly introducing switching costs (as in [10]), or by assuming a kind of inertia in the switching mechanism. With the latter approach, only a fraction of individual agents decide to reconsider their market choice at each time step. Applications can be found in [20] and in [21]. Preliminary simulations of our model confirm our intuition that inertia does exert a stabilizing impact on the dynamics.

\footnotetext{
${ }^{19}$ Dieci and Westerhoff [18] provide a preliminary discussion of the case where the two goods are complements, or substitutes, in a related model based upon linear demand and supply.
} 
Third, our framework might be useful to establish connections with certain experimental literature. Contributions which seem relevant to the paper include [22] and [23]. The former studies an experiment where suppliers have to choose repeatedly between two different locations to supply their commodity, and resulting dynamic patterns for quantities supplied reveal cyclical fluctations similar to those presented here. The latter investigates a coordination game dealing with a similar type of problem. The theoretical model advanced here might contribute to explaining some of the experimental results from those papers. Also the 'El Farol bar problem' ([24]) and the 'minority game' ([25]) seem to be related to the model studied in the present paper.

Fourth, an important question is the extent to which the cyclical and even chaotic dynamics generated by the model depend on the assumption of naïve (or, more generally, adaptive) expectations. A more general perspective should take into account, for instance, the role played by forward-looking agents, along the lines of [26].

Fifth, the model proposed here may also be relevant from a policy perspective. It is obvious that policy makers planning stabilization schemes in one market should pay great attention to the way in which they will influence the overall system of interacting markets. The effect of different types of regulatory interventions on our results would be an interesting question for future research.

Acknowledgements The authors would like to thank two anonymous referees for their valuable comments, which helped to greatly improve this paper. The usual caveat applies. R. Dieci acknowledges the support given by MIUR in project PRIN-2004137559: "Nonlinear models in economics and finance: interactions, complexity and forecasting." 


\section{References}

[1] M. Ezekiel, The cobweb theorem, Quart. J. Econ. 52 (1938) 255-280.

[2] P. Cashin, J. McDermott, A. Scott, Booms and slumps in world commodity prices, J. Devel. Econ. 69 (2002) 277-296.

[3] S. Baak, Test for bounded rationality with a linear dynamic model distorted by heterogeneous expectations, J. Econ. Dynam. Control 23 (1999) 1517-1543.

[4] J. Chavas, On information and market dynamics: The case of the U.S. beef market, J. Econ. Dynam. Control 24 (2000) 833-853.

[5] J. Sonnemans, C.H. Hommes, J. Tuinstra, H. van de Velden, The instability of a heterogeneous cobweb economy: A strategy experiment on expectation formation, J. Econ. Behav. Org. 54 (2004) 453-481.

[6] C. Chiarella, The cobweb model, its instability and the onset of chaos, Econ. Model. 5 (1988) $377-384$.

[7] R.H. Day, Complex economic dynamics: An introduction to dynamical systems and market mechanisms, MIT Press, Cambridge, MA, 1994.

[8] C.H. Hommes, Dynamics of the cobweb model with adaptive expectations and non-linear supply and demand, J. Econ. Behav. Org. 24 (1994) 315-335.

[9] C.H. Hommes, On the consistency of backward-looking expectations: The case of the cobweb, J. Econ. Behav. Org. 33 (1998) 333-362.

[10] W.A. Brock, C.H. Hommes, A rational route to randomness, Econometrica 65 (1997) 10591095.

[11] J. Goeree, C.H. Hommes, Heterogeneous beliefs and the non-linear cobweb model, J. Econ. Dynam. Control 24 (2000) 761-798.

[12] W. Branch, Local convergence properties of a cobweb model with rationally heterogeneous expectations, J. Econ. Dynam. Control 27 (2002) 63-85.

[13] C. Chiarella, X.-Z. He, Dynamics of beliefs and learning under $a_{L^{-}}$processes - the heterogeneous case, J. Econ. Dynam. Control 27 (2003) 503-532. 
[14] J.-M. Boussard, When risk generates chaos, J. Econ. Behav. Org. 29 (1996) 433-446.

[15] C. Chiarella, X.-Z. He, H. Hung, P. Zhu, An analysis of the cobweb model with bounded rational heterogeneous producers, J. Econ. Behav. Org. 61 (2006) 750-768.

[16] M. Currie, I. Kubin, Non-linearities and partial analysis, Econ. Lett. 49 (1995) 27-31.

[17] C.H. Hommes, A. van Eekelen, Partial equilibrium analysis in a noisy chaotic market, Econ. Lett. 53 (1996) 275-282.

[18] R. Dieci, F. Westerhoff, Interacting cobweb markets, Working Paper, University of Bologna, 2009 .

[19] S.P. Anderson, A. de Palma, J.-F. Thisse, Discrete Choice Theory of Product Differentiation, MIT Press, Cambridge, MA, 1992.

[20] C. Diks, R. van der Weide, Herding, a-synchronous updating and heterogeneity in memory in a CBS, J. Econ. Dynam. Control 29 (2005) 741-763.

[21] C.H. Hommes, H. Huang, D. Wang, A robust rational route to randomness in a simple asset pricing model, J. Econ. Dynam. Control 29 (2005) 1043-1072.

[22] D.J. Meyer, J.B. Van Huyck, R.C. Battalio, T.R. Saving, History's role in coordinating decentralized allocation decisions, J. Polit. Economy 100 (1992) 292-316.

[23] J. Ochs, The coordination problem in decentralized markets: an experiment, Quart. J. Econ. 105 (1990) 545-559.

[24] W.B. Arthur, Inductive reasoning and bounded rationality, Amer. Econ. Rev. 84 (1994) $406-411$.

[25] D. Challet, Y.-C. Zhang, Emergence of cooperation and organization in an evolutionary game, Physica A 246 (1997) 407-418.

[26] W.A. Brock, P. Dindo, C.H. Hommes, Adaptive rational equilibrium with forward looking agents, Int. J. Econ. Theory 2 (2006) 241-278.

[27] G. Gandolfo, Economic Dynamics (3rd edition), Springer, Berlin, 1996. 


\section{Appendix A: Proof of Proposition 1}

(i) Assume that a steady state exists. Steady-state prices and quantities, $\bar{P}_{X}, \bar{P}_{Z}, \bar{S}_{X}, \bar{S}_{Z}$, and steady-state profits, $\bar{\pi}_{X}$ and $\bar{\pi}_{Z}$, must then satisfy the following set of conditions ${ }^{20}$ :

$$
\begin{gathered}
\bar{P}_{X}=D_{X}^{-1}\left(\frac{N}{2}(1+\bar{\Omega}) G_{X}\left(\bar{P}_{X}\right)\right), \quad \bar{P}_{Z}=D_{Z}^{-1}\left(\frac{N}{2}(1-\bar{\Omega}) G_{Z}\left(\bar{P}_{Z}\right)\right) \\
\bar{S}_{X}=G_{X}\left(\bar{P}_{X}\right), \quad \bar{S}_{Z}=G_{Z}\left(\bar{P}_{Z}\right) \\
\bar{\pi}_{X}=\bar{P}_{X} \bar{S}_{X}-C_{X}\left(\bar{S}_{X}\right), \quad \bar{\pi}_{Z}=\bar{P}_{Z} \bar{S}_{Z}-C_{Z}\left(\bar{S}_{Z}\right)
\end{gathered}
$$

where $\bar{\Omega}$ represents the stationary "distribution" of producers across markets, satisfying

$$
\bar{\Omega}=\tanh \left[\frac{f}{2}\left(\bar{\pi}_{X}-\bar{\pi}_{Z}\right)\right]
$$

Due to equation (29) it will not be possible, in general (except in the case $f=0$ ), to solve for stationary values explicitly, even under simple specifications of $D_{X}, D_{Z}, C_{X}, C_{Z}$. In order to show that a steady state does exist and is unique, it is convenient to treat $\bar{\Omega}$ as parametric, first, and to regard equilibrium prices (26), quantities (27), and profits (28) as functions of stationary distribution $\bar{\Omega}$, similarly to the fixed-proportion case discussed in Section 2 (see equation (7)). In particular, by our assumptions on demand and supply curves, equilibrium prices $\bar{P}_{X}=\bar{P}_{X}(\bar{\Omega})$ and $\bar{P}_{Z}=\bar{P}_{Z}(\bar{\Omega})$ are uniquely defined implicitly as functions of $\bar{\Omega}(-1<\bar{\Omega}<1)$ by conditions (26), whereas steady-state quantities and profits are functions of $\bar{\Omega}$ via $\bar{P}_{X}$ and $\bar{P}_{Z}$. We can then compute the derivatives of $\bar{\pi}_{X}(\bar{\Omega})$ and $\bar{\pi}_{Z}(\bar{\Omega})$. First of all, we obtain

$$
\frac{d \bar{\pi}_{X}}{d \bar{\Omega}}=\frac{d \bar{P}_{X}}{d \bar{\Omega}}\left[\bar{S}_{X}+G_{X}^{\prime}\left(\bar{P}_{X}\right)\left(\bar{P}_{X}-C_{X}^{\prime}\left(\bar{S}_{X}\right)\right)\right]=\frac{d \bar{P}_{X}}{d \bar{\Omega}} \bar{S}_{X}
$$

where the latter simplification is possible because $\bar{S}_{X}=G_{X}\left(\bar{P}_{X}\right):=\left(C_{X}^{\prime}\right)^{-1}\left(\bar{P}_{X}\right)$. In a similar manner, one obtains

$$
\frac{d \bar{\pi}_{Z}}{d \bar{\Omega}}=\frac{d \bar{P}_{Z}}{d \bar{\Omega}} \bar{S}_{Z}
$$

Furthermore, differentiation of implicit functions $\bar{P}_{X}(\bar{\Omega})$ and $\bar{P}_{Z}(\bar{\Omega})$ yields

$$
\frac{d \bar{P}_{X}}{d \bar{\Omega}}=-\frac{\left(D_{X}^{-1}\right)^{\prime}\left(\bar{S}_{X}^{A}\right) \frac{N}{2} G_{X}\left(\bar{P}_{X}\right)}{\left(D_{X}^{-1}\right)^{\prime}\left(\bar{S}_{X}^{A}\right) \frac{N}{2}(1+\bar{\Omega}) G_{X}^{\prime}\left(\bar{P}_{X}\right)-1}=-\frac{\frac{N}{2} G_{X}\left(\bar{P}_{X}\right)}{\frac{N}{2}\left(1+\bar{\Omega}^{\prime}\right) G_{X}^{\prime}\left(\bar{P}_{X}\right)-D_{X}^{\prime}\left(\bar{P}_{X}\right)},
$$

\footnotetext{
${ }^{20}$ Such conditions are obtained by imposing $\left(P_{X, t-1}, P_{Z, t-1}, S_{X, t-1}, S_{Z, t-1}\right)=\left(P_{X, t}, P_{Z, t}, S_{X, t}, S_{Z, t}\right)=$ $\left(\bar{P}_{X}, \bar{P}_{Z}, \bar{S}_{X}, \bar{S}_{Z}\right)$ in equations (12)-(17).
} 
and

$$
\frac{d \bar{P}_{Z}}{d \bar{\Omega}}=\frac{\left(D_{Z}^{-1}\right)^{\prime}\left(\bar{S}_{Z}^{A}\right) \frac{N}{2} G_{Z}\left(\bar{P}_{Z}\right)}{\left(D_{Z}^{-1}\right)^{\prime}\left(\bar{S}_{Z}^{A}\right) \frac{N}{2}(1-\bar{\Omega}) G_{Z}^{\prime}\left(\bar{P}_{Z}\right)-1}=\frac{\frac{N}{2} G_{Z}\left(\bar{P}_{Z}\right)}{\frac{N}{2}(1-\bar{\Omega}) G_{Z}^{\prime}\left(\bar{P}_{Z}\right)-D_{Z}^{\prime}\left(\bar{P}_{Z}\right)},
$$

where

$$
\bar{S}_{X}^{A}:=\frac{N}{2}(1+\bar{\Omega}) G_{X}\left(\bar{P}_{X}\right), \quad \bar{S}_{Z}^{A}:=\frac{N}{2}(1-\bar{\Omega}) G_{Z}\left(\bar{P}_{Z}\right)
$$

are total quantities supplied in the two markets at the steady state, and where $\left(D_{X}^{-1}\right)^{\prime}\left(\bar{S}_{X}^{A}\right)=$ $1 / D_{X}^{\prime}\left(\bar{P}_{X}\right),\left(D_{Z}^{-1}\right)^{\prime}\left(\bar{S}_{Z}^{A}\right)=1 / D_{Z}^{\prime}\left(\bar{P}_{Z}\right)$ by inverse-function differentiation rule. Since $D_{X}^{\prime}<0$, $D_{Z}^{\prime}<0, G_{X}^{\prime}>0, G_{Z}^{\prime}>0$, it follows that for any $\bar{\Omega}$

$$
\frac{d \bar{P}_{X}}{d \bar{\Omega}}<0, \quad \frac{d \bar{P}_{Z}}{d \bar{\Omega}}>0, \quad \frac{d \bar{\pi}_{X}}{d \bar{\Omega}}<0, \quad \frac{d \bar{\pi}_{Z}}{d \bar{\Omega}}>0
$$

and therefore

$$
\frac{d}{d \bar{\Omega}}\left(\bar{\pi}_{X}-\bar{\pi}_{Z}\right)<0
$$

This proves that the right-hand side of $(29)$ - denoted by $\psi(\bar{\Omega})$ - is a strictly decreasing function of $\bar{\Omega}$.

The stationary distribution $\bar{\Omega}$, in turn, is determined endogenously by equation (29), which can be rewritten as

$$
\psi(\bar{\Omega})-\bar{\Omega}=0 .
$$

Since $-1<\tanh (y)<1$ for any $y$, one obtains $1>\psi(-1)>\psi(1)>-1$. It follows that equation (32) admits a unique solution in the interval $[-1,1]$. This proves that a unique steady state exists.

(ii) Coming to the sign of $\bar{\Omega}$, note first that $\bar{\Omega}=0$ when $f=0$ (independent markets) and that the steady-state profits in this case are given by

$$
\bar{\pi}_{X}^{0}:=\bar{P}_{X}^{0} G_{X}\left(\bar{P}_{X}^{0}\right)-C_{X}\left(G_{X}\left(\bar{P}_{X}^{0}\right)\right), \quad \bar{\pi}_{Z}^{0}:=\bar{P}_{Z}^{0} G_{Z}\left(\bar{P}_{Z}^{0}\right)-C_{Z}\left(G_{Z}\left(\bar{P}_{Z}^{0}\right)\right)
$$

where $\bar{P}_{X}^{0}$ and $\bar{P}_{Z}^{0}$ are implicitly defined, respectively, by

$$
D_{X}^{-1}\left(\frac{N}{2} G_{X}\left(\bar{P}_{X}^{0}\right)\right)-\bar{P}_{X}^{0}=0, \quad D_{Z}^{-1}\left(\frac{N}{2} G_{Z}\left(\bar{P}_{Z}^{0}\right)\right)-\bar{P}_{Z}^{0}=0 .
$$

Assume now $f>0$ and note that, from (30), $\bar{\pi}_{X}<\bar{\pi}_{X}^{0}$ and $\bar{\pi}_{Z}>\bar{\pi}_{Z}^{0}$ for $\bar{\Omega}>0$, whereas the reverse inequalities hold for $\bar{\Omega}<0$. From (29), $\bar{\Omega}$ has the same sign of the steady-state profit 
differential, $\bar{\pi}_{X}-\bar{\pi}_{Z}$, since $\tanh (y) \gtreqless 0$ for $y \gtreqless 0$. It follows that (29) cannot hold with $\bar{\pi}_{X}^{0} \leq \bar{\pi}_{Z}^{0}$ and $\bar{\Omega}>0$, i.e $\bar{\Omega}>0 \Rightarrow \bar{\pi}_{X}^{0}>\bar{\pi}_{Z}^{0}$. Similarly one can check that (29) cannot be satisfied with $\bar{\pi}_{X}^{0}>\bar{\pi}_{Z}^{0}$ and $\bar{\Omega} \leq 0$, i.e. $\bar{\pi}_{X}^{0}>\bar{\pi}_{Z}^{0} \Rightarrow \bar{\Omega}>0$. Therefore $\bar{\Omega}>0 \Leftrightarrow \bar{\pi}_{X}^{0}>\bar{\pi}_{Z}^{0}$. In a similar manner, it can be proven that $\bar{\Omega}<0 \Leftrightarrow \bar{\pi}_{X}^{0}<\bar{\pi}_{Z}^{0}$.

With regard to the dependence of $\bar{\Omega}$ on the 'intensity of choice' parameter $f$, we set

$$
\Gamma(f, \bar{\Omega}):=\tanh \left[\frac{f}{2}\left(\bar{\pi}_{X}-\bar{\pi}_{Z}\right)\right]-\bar{\Omega}
$$

where $\bar{\pi}_{X}$ and $\bar{\pi}_{Z}$ are defined by (28). Again from (29), rewritten as $\Gamma(f, \bar{\Omega})=0$, one obtains

$$
\frac{d \bar{\Omega}}{d f}=-\frac{\frac{\partial \Gamma}{\partial f}}{\frac{\partial \Gamma}{\partial \bar{\Omega}}},
$$

where

$$
\begin{gathered}
\frac{\partial \Gamma}{\partial f}=\left[1-\tanh ^{2}\left(\frac{f}{2}\left(\bar{\pi}_{X}-\bar{\pi}_{Z}\right)\right)\right] \frac{1}{2}\left(\bar{\pi}_{X}-\bar{\pi}_{Z}\right) \\
\frac{\partial \Gamma}{\partial \bar{\Omega}}=\left[1-\tanh ^{2}\left(\frac{f}{2}\left(\bar{\pi}_{X}-\bar{\pi}_{Z}\right)\right)\right] \frac{f}{2}\left(\frac{d}{d \bar{\Omega}}\left(\bar{\pi}_{X}-\bar{\pi}_{Z}\right)\right)-1 .
\end{gathered}
$$

Due to (31), the partial derivative $\frac{\partial \Gamma}{\partial \bar{\Omega}}$ is negative for any $\bar{\Omega}$, from which it follows that $\frac{d \bar{\Omega}}{d f}$ has the same sign of $\frac{\partial \Gamma}{\partial f}$, i.e. of the steady-state profit differential $\left(\bar{\pi}_{X}-\bar{\pi}_{Z}\right)$. The latter quantity, in turn, has the same sign of $\bar{\Omega}$. Therefore

$$
\frac{d \bar{\Omega}}{d f} \gtreqless 0 \Longleftrightarrow \bar{\Omega} \gtreqless 0
$$

Finally, for $f=0$ (in which case $\bar{\Omega}=0$ ), one gets

$$
\left.\frac{d \bar{\Omega}}{d f}\right|_{f=0}=\frac{1}{2}\left(\bar{\pi}_{X}^{0}-\bar{\pi}_{Z}^{0}\right) .
$$

It follows that if $\bar{\pi}_{X}^{0}>\bar{\pi}_{Z}^{0}\left(\bar{\pi}_{X}^{0}<\bar{\pi}_{Z}^{0}\right)$, steady-state distribution $\bar{\Omega}$ is a strictly increasing (decreasing) function of parameter $f$, for $f$ ranging from zero to infinity. 


\section{Appendix B: Proof of Proposition 2}

(i) The Jacobian matrix at the steady state (denote it by $J$ ) can be rewritten as a function of steady-state distribution $\bar{\Omega}$. Note first that $J$ has the following lower block triangular structure

$$
J=\left[\begin{array}{ll}
\mathbf{A} & \mathbf{0} \\
\mathbf{C} & \mathbf{0}
\end{array}\right]
$$

where $\mathbf{A}, \mathbf{C}$, are two-dimensional blocks, while $\mathbf{0}$ denotes the two-dimensional null matrix. A null block occupies the upper right corner because $F_{X}$ and $F_{Z}$, defined by (12) and (13), depend on $S_{X, t-1}$ and $S_{Z, t-1}$ only via $\Omega_{t}$ (as defined in eq. (16)), where $\partial \Omega / \partial S_{X}, \partial \Omega / \partial S_{Z}$ include the factors $\left(P_{X}-C_{X}^{\prime}\left(S_{X}\right)\right),\left(P_{Z}-C_{Z}^{\prime}\left(S_{Z}\right)\right)$, respectively. The latter quantities vanish at the steady state, due to (27) and the fact that $G_{X}:=\left(C_{X}^{\prime}\right)^{-1}, G_{Z}:=\left(C_{Z}^{\prime}\right)^{-1}$. Furthermore, block A can be written as follows

$$
\mathbf{A}=\left[\begin{array}{cc}
\frac{N(1+\bar{\Omega})}{2 D_{X}^{\prime}\left(\bar{P}_{X}\right)}\left[G_{X}^{\prime}\left(\bar{P}_{X}\right)+(1-\bar{\Omega}) \frac{f}{2} \bar{S}_{X}^{2}\right] & -\frac{N}{2 D_{X}^{\prime}\left(\bar{P}_{X}\right)} \frac{f}{2}\left(1-\bar{\Omega}^{2}\right) \bar{S}_{X} \bar{S}_{Z} \\
-\frac{N}{2 D_{Z}^{\prime}\left(\bar{P}_{Z}\right)} \frac{f}{2}\left(1-\bar{\Omega}^{2}\right) \bar{S}_{X} \bar{S}_{Z} & \frac{N(1-\bar{\Omega})}{2 D_{Z}^{\prime}\left(\bar{P}_{Z}\right)}\left[G_{Z}^{\prime}\left(\bar{P}_{Z}\right)+\left(1+\bar{\Omega}_{2} \frac{f}{2} \bar{S}_{Z}^{2}\right]\right.
\end{array}\right]
$$

The reason for this simplified form is that the partial derivatives of $\Omega$ with respect to the state variables include the factor $\left[1-\tanh ^{2}\left(\frac{f}{2}\left(\pi_{X}-\pi_{Z}\right)\right)\right]$, which becomes equal to $\left(1-\bar{\Omega}^{2}\right)$ at the steady state, where $\bar{\Omega}=\tanh \left[\frac{f}{2}\left(\bar{\pi}_{X}-\bar{\pi}_{Z}\right)\right]$, according to (29).

The structure of $J$ implies that two eigenvalues are zero, whereas the remaining eigenvalues are those of the two-dimensional matrix $\mathbf{A}$. In order to simplify the notation, define aggregate quantities $\gamma_{X}, \gamma_{Z}, \delta_{X}, \delta_{Z}$ according to (19)-(20), and denote by $\operatorname{Tr}$ and Det the trace and the determinant of $\mathbf{A}$, namely:

$$
\begin{gathered}
\operatorname{Tr}=-\left[\gamma_{X}\left(1+f \delta_{X}\right)+\gamma_{Z}\left(1+f \delta_{Z}\right)\right]<0 \\
\operatorname{Det}=\gamma_{X} \gamma_{Z}\left(1+f \delta_{X}\right)\left(1+f \delta_{Z}\right)-\gamma_{X} \gamma_{Z} f^{2} \delta_{X} \delta_{Z}=\gamma_{X} \gamma_{Z}\left[1+f\left(\delta_{X}+\delta_{Z}\right)\right]>0 .
\end{gathered}
$$

From the characteristic polynomial of $\mathbf{A}, \mathcal{P}(\lambda):=\lambda^{2}-\operatorname{Tr} \lambda+$ Det, it can be seen that

$$
\operatorname{Tr}^{2}-4 \operatorname{Det}=\left[\gamma_{X}\left(1+f \delta_{X}\right)-\gamma_{Z}\left(1+f \delta_{Z}\right)\right]^{2}+4 f^{2} \gamma_{X} \gamma_{Z} \delta_{X} \delta_{Z} \geq 0
$$

and therefore the eigenvalues of $\mathbf{A}$ are real.

The region of local asymptotic stability of the steady state is defined, in general, by the 
following set of inequalities in the plane $\operatorname{Tr}$, Det.

$$
1-\operatorname{Tr}+\operatorname{Det}>0, \quad 1+\operatorname{Tr}+\operatorname{Det}>0, \quad 1-\operatorname{Det}>0 .
$$

As is well known, (35) provides a necessary and sufficient condition for both eigenvalues to be inside the unit circle of the complex plane (see, e.g. [27]). If the inequalities (35) are simultaneously satisfied for a given parameter configuration, and boundary $1-\operatorname{Tr}+\operatorname{Det}=0$ is crossed when a critical parameter is varied, then one of the two eigenvalues becomes larger than +1 (which may result, for instance, in a saddle-node bifurcation). Similarly, crossing boundary $1+\operatorname{Tr}+\operatorname{Det}=0$ entails a bifurcation with one eigenvalue equal to -1 (Flip bifurcation), while along boundary $D e t=1$ the eigenvalues are complex conjugate with unit modulus (and crossing the boundary results in a Neimark-Sacker bifurcation). However, for the particular case at hand, where $\operatorname{Tr}<0$ and $\operatorname{Det}>0$, the first inequality in (35) is always true, which rules out the possibility of a bifurcation of the first type discussed above. Moreover, we know that the eigenvalues are real for any selection of parameters, due to (34), which excludes the possibility of Neimark-Sacker bifurcation. Therefore, by taking into account the additional restrictions discussed above, the stability region (35) reduces to

$$
\operatorname{Tr}>-2,1+\operatorname{Tr}+\operatorname{Det}>0
$$

as can be easily checked. ${ }^{21}$ The two inequalities in (36) can be rewritten, respectively, as

$$
\begin{gathered}
\gamma_{X}\left(1+f \delta_{X}\right)+\gamma_{Z}\left(1+f \delta_{Z}\right)<2, \\
{\left[1-\gamma_{X}\left(1+f \delta_{X}\right)\right]\left[1-\gamma_{Z}\left(1+f \delta_{Z}\right)\right]>f^{2} \gamma_{X} \gamma_{Z} \delta_{X} \delta_{Z},}
\end{gathered}
$$

or in the equivalent form (18), and stability can generically be lost only via a Flip bifurcation when one of the eigenvalues exceeds -1 , which violates (38). ${ }^{22}$

(ii) Since $(38)$ implies $\left[1-\gamma_{X}\left(1+f \delta_{X}\right)\right]\left[1-\gamma_{Z}\left(1+f \delta_{Z}\right)\right]>0$, the two inequalities (37)-(38) together imply

$$
\gamma_{X}\left(1+f \delta_{X}\right)<1, \quad \gamma_{Z}\left(1+f \delta_{Z}\right)<1,
$$

\footnotetext{
${ }^{21}$ Note that condition $-2<\operatorname{Tr}<0$, together with the fact that eigenvalues are real $\left(T r^{2}-4 D e t \geq 0\right)$, implies in particular Det $<1$.

${ }^{22}$ The case in which stability is lost by violation of condition $\operatorname{Tr}>-2$ (i.e. (37)) is nongeneric. Moving from the interior of the stability region in the plane $(T r, D e t)$, the violation of such a condition can only occur through point $(-2,1)$, which implies the simultaneous violation of condition $1+\operatorname{Tr}+\operatorname{Det}>0$ (i.e. condition (38)).
} 
or equivalently, using the original parameters, (21)-(22). The latter therefore represents a necessary condition for local asymptotic stability.

\section{Appendix C: Proof of the Corollaries}

In order to prove Corollary 3 , note that in the constant-proportion model with $N \bar{W}_{X}=$ $N(1+\bar{\Omega}) / 2$ producers in market $X$ and $N \bar{W}_{Z}=N(1-\bar{\Omega}) / 2$ producers in market $Z$, the local stability conditions of the two markets are given by (10), or equivalently

$$
\gamma_{X}<1, \quad \gamma_{Z}<1
$$

It is clear that, for $f>0$, the local stability condition (37)-(38) of the full model implies (40), via (39). In contrast, if (40) is satisfied (both markets are stable in the related fixed-proportion model) but $\gamma_{X}$, or $\gamma_{Z}$ are large enough, necessary condition (39) for the stability of the complete model will be violated.

In order to prove Corollary 4, note first that the necessary stability condition (39) (or equivalently (21)-(22)) further implies the following inequality to hold at the steady state of the complete model:

$$
\left|\frac{N}{2}(1+\bar{\Omega}) \frac{G_{X}^{\prime}\left(\bar{P}_{X}\right)}{D_{X}^{\prime}\left(\bar{P}_{X}\right)}\right|<1 .
$$

Suppose, without loss of generality, that $\bar{\pi}_{X}^{0}>\bar{\pi}_{Z}^{0}$, i.e. market $X$ has a higher steady-state profit in the absence of switching and independent markets $(f=0)$. Then Proposition 1 ensures that $\bar{\Omega}>0$ for any $f>0$. Therefore, it follows from (30) that $\bar{P}_{X}^{0}:=\bar{P}_{X}(0)>\bar{P}_{X}$, where $\bar{P}_{X}^{0}$ (defined by (33)) denotes the steady state price in market $X$ in the absence of switching $(f=0$ and $\bar{\Omega}=0)$. If demand and (individual) supply curves in market $X$ are such that the ratio $\left|G_{X}^{\prime} / D_{X}^{\prime}\right|$ between their slopes decreases or remains constant when the price changes from $\bar{P}_{X}$ to $\bar{P}_{X}^{0}$, namely if

$$
\left|\frac{G_{X}^{\prime}\left(\bar{P}_{X}^{0}\right)}{D_{X}^{\prime}\left(\bar{P}_{X}^{0}\right)} \frac{D_{X}^{\prime}\left(\bar{P}_{X}\right)}{G_{X}^{\prime}\left(\bar{P}_{X}\right)}\right| \leq 1
$$

then (41) implies

$$
\left|\frac{N}{2} \frac{G_{X}^{\prime}\left(\bar{P}_{X}^{0}\right)}{D_{X}^{\prime}\left(\bar{P}_{X}^{0}\right)}\right|<1,
$$

i.e. the condition for stability of the isolated market $X$. Condition (42) is satisfied, for instance, when demand and supply are linear or concave, as well as for a number of cases where demand 
is convex. ${ }^{23}$ A symmetric reasoning holds for the case where $\bar{\pi}_{X}^{0}<\bar{\pi}_{Z}^{0}$, and therefore $\bar{\Omega}<0$. In other words, for a broad class of demand and supply curves, the local stability of the steady state of the full model with interacting markets cannot subsist without stability of at least one of the two markets, namely, that which would be more profitable at the steady state, in the case of zero intensity of switching.

\footnotetext{
${ }^{23}$ Moreover, condition (42) is only sufficient, and the implication stated by Corollary 4 may still be true under more general demand and supply curves.
} 


\section{Figure captions}

\section{Figure 1}

The first three panels show the price in market $X$, the price in market $Z$, and the distribution of producers in the time domain. The parameter setting is as in Section 3.2. The bottom two panels show the dynamics in phase space, after omitting a long transient phase (10000 iterations), by means of projections of the attractor on plane $P_{X}, P_{Z}$ and on plane $P_{X}, S_{X}$, respectively.

\section{Figure 2}

Bifurcation diagrams versus parameter $f$. The first panel represents the price in market $X$, whereas the second panel displays the proportion of producers in market $X$ after omitting a transient of 10000 iterations. The parameter is increased in 1000 discrete steps, in the range $[0,0.7]$. The remaining parameters are as in figure 1 . 
Figure 1
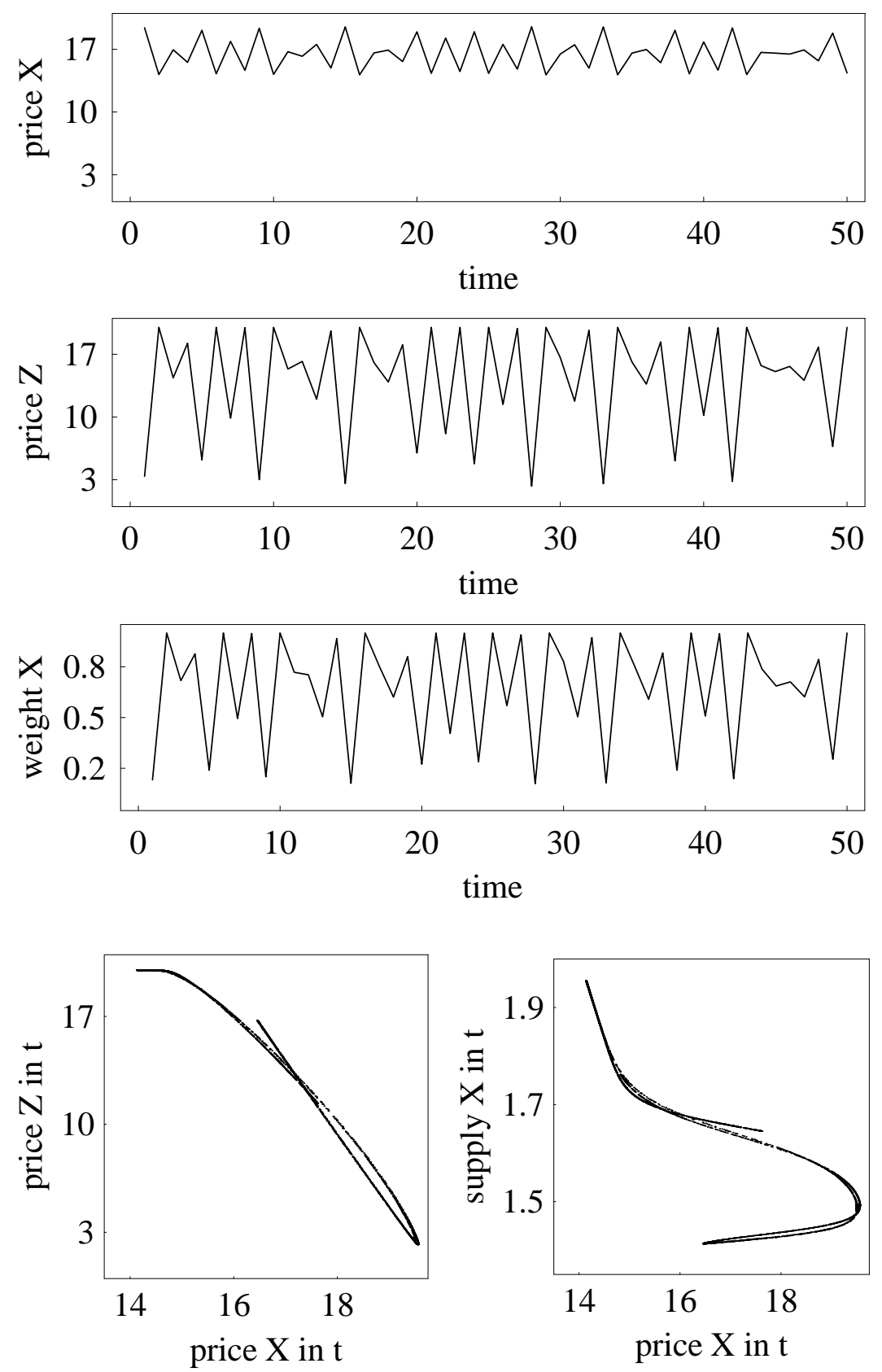
Figure 2
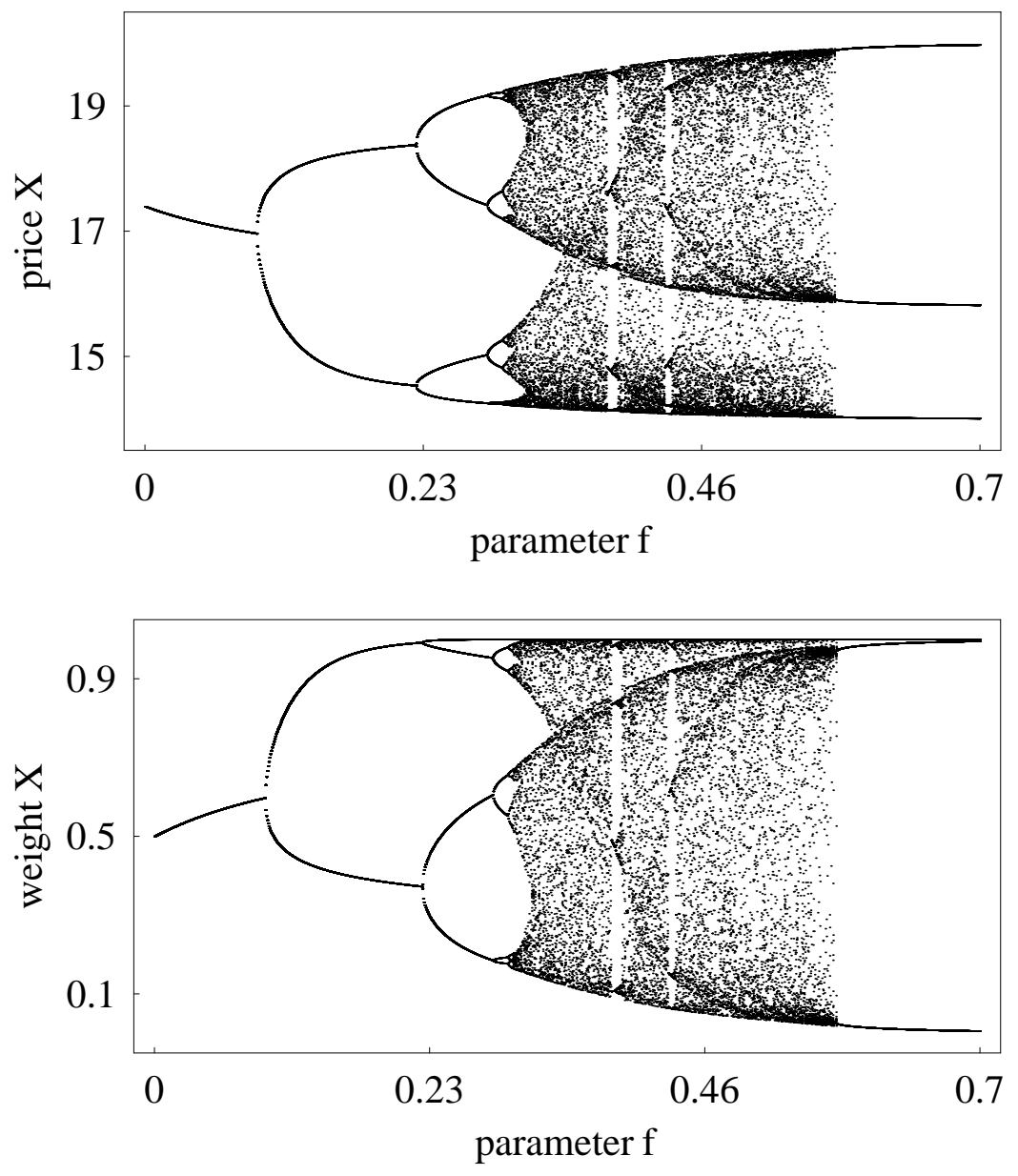